\title{
Simulation and analysis of frequency modulation circuit based on LM566
}

\author{
Zhang Qian ${ }^{1,}$, , Hu Meng ${ }^{2, b}$ \\ ${ }^{1}$ Zhenjiang Watercraft College, Zhenjiang, 212003, Jiangsu Province, China \\ ${ }^{2}$ Zhenjiang Watercraft College, Zhenjiang, 212003, Jiangsu Province, China \\ aemail: zhangqian_zj@sina.com
}

Keywords: Simulation; Voltage-Controlled Oscillator; Frequency Modulation; Electronic Circuit

\begin{abstract}
LM566 is a monolithic integration VCO chip of integral-Schmitt Trigger Circuit type. It is the modulator and demodulator in teaching of FM circuit. Its operating features can be displayed vividly on software platform through simulation the chip to facilitate quantitative analysis. As there is no model for LM566 in component library, basic component models existing Multisim component library are used to constitute internal structure of LM566 chip to solve the problem. By analyzing static operating mode and dynamic operating mode of peripheral FM circuit, simulation result and testing result indicate that chip model in the thesis can objectively describe the basic characteristics of LM566, which lays the foundation for simulation analysis of all functional circuits including FM circuit via the chip and effectively extends application scope of Multisim simulation software. It has great application value.
\end{abstract}

\section{Introduction}

Electronic Design Automation (EDA) technology makes it possible for design personnel of electronic circuit to complete function design, logic design, performance analysis and time sequence test of the circuit in computer until the automatic generation of printed circuit board, which includes temperature distribution and electromagnetic compatibility of printed board. It represents the technical trend of electronic system design. The thesis precisely realizes modeling of integrated voltage-controlled oscillator chip LM566 in Multisim12 simulation environment and simulation analysis of FM circuit performance through study and trials. Simulation and test results indicate the model describes the basic characteristics of the chip objectively and precisely.

\section{Main Functions and Features of Multisim12}

Multisim12 is a simulation tool based on Windows that is released by National Instruments. It applies to design of board-level analog/digital circuit board. [1]The software includes graphic input of schematic circuit diagram and input mode of hardware description language. It has rich simulation analysis capacities and can design and verify the circuit quickly, easily and efficiently by combining intuitive capture and powerful simulation. User can create circuit diagram with complete component library instantly and imitate circuit behavior through industrial standard SPICE simulator.

Multisim12 has the following features:

1) Combination of system-level closed loop simulation of Analog and digital application with Multisim and LabVIEW can save time effectively in the design process. [2]It can not only simulate analog circuit or digital circuit respectively but also implement mixed-signal simulation, especially simulation of newly increased radio frequency (RF) circuit. Error information will be displayed when simulation fails to prompt possible reasons for error. Simulation result can be saved and printed at any moment;

2) Brand new database improves new electromechanical model, AC/DC power converter and switch mode power used in design power application;

3) More than 2,000 new database components from semiconductor manufacturers of Analog 
Devices, National Semiconductor, NXP and Philips;

4) More than 90 new pin-precise connections, which makes the design of customized accessory of NI hardware easier.

\section{FM Circuit Based on LM566}

Purpose of the design is to further understand voltage-controlled oscillator and the theory to constitute frequency modulation through it, profoundly grasp operating pattern of integrated circuit frequency modulator, extend Multisim application scope and improve software application capacity by modeling the LM566 chip in Multisim simulation environment.

\section{Introduction to LM566 chip}

Lm566 is a monolithic integration VCO circuit of integral-Schmitt Trigger Circuit type. Where, (8) pin-connected positive power (1)pin-connected negative power (or ground) (2)suspended pin (3) pin-output square waves (4)pin-output triangular waves (5) pin-connected input voltage (6) pin-connected timed resistance (7) pin-connected timed capacitance. [3]

\section{Operating theory of hardware circuit}

Fig. 1 is schematic diagram of frequency circuit. The power supply of the system is subject to $+5 \mathrm{~V}$ and $-5 \mathrm{~V}$ currents, which connect pin (8) and pin (1) respectively. $R_{3}$ and $R_{p} 1$ constitute timed capacitance, value of timed capacitance can be changed by regulating $R_{p} 1$. Input (pin (5)) has two operating modes: one is static operating mode (pin (5) connects the switch of circuit where C2 is). Direct current is input through pin (5) and the output is un-modulated carrier wave; regulate $R_{p} 1$ to change carrier wave frequency (cycle). Second is dynamic operating mode (switch corresponding to pin (5), switch at break and both switches connected). Superpose modulating signal input from IN terminal and DC distribution of $R_{6}$ on pin (5) so that the output is modulated wave. [4] The carrier wave acquired by the design at $R_{1}$ is non-sine modulated wave of square wave. Triangular wave output can be acquired at $R_{2}$ (pin (4)). Calculation formula of modulated frequency is $f=\frac{2\left(V_{8}-V_{5}\right)}{R_{T} C_{T} V_{8}}(\mathrm{HZ})$

\section{Construction of multisim simulation platform}

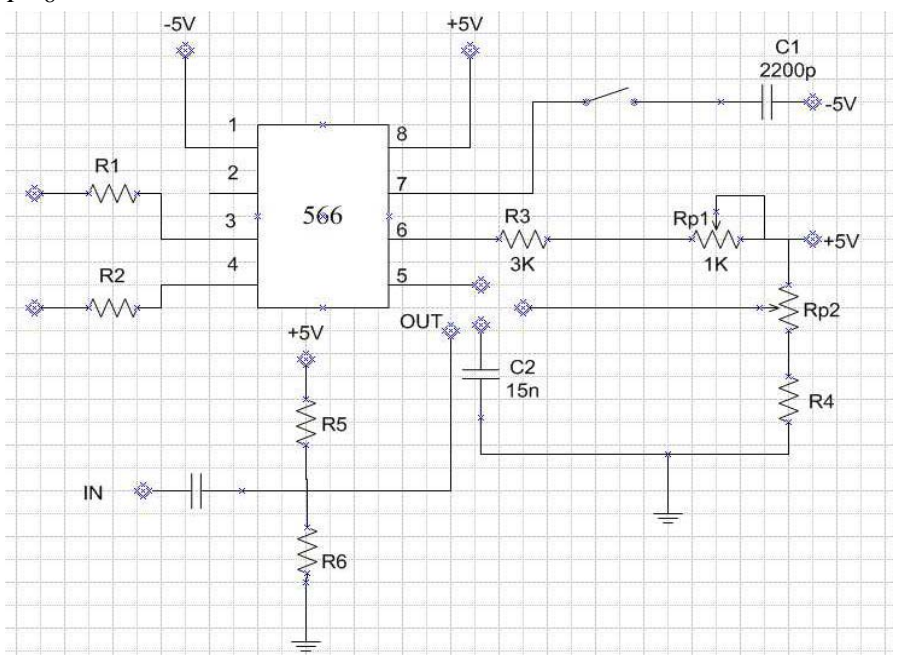

Fig.1. Schematic diagram of FM circuit

As there is no LM566 in component library in Multisim12 simulation software, simulation model of chip must be constituted in Multisim12. According to Datasheet provided by chip manufacturer, LM566 Multisim simulation model is created as shown in Fig.2. 


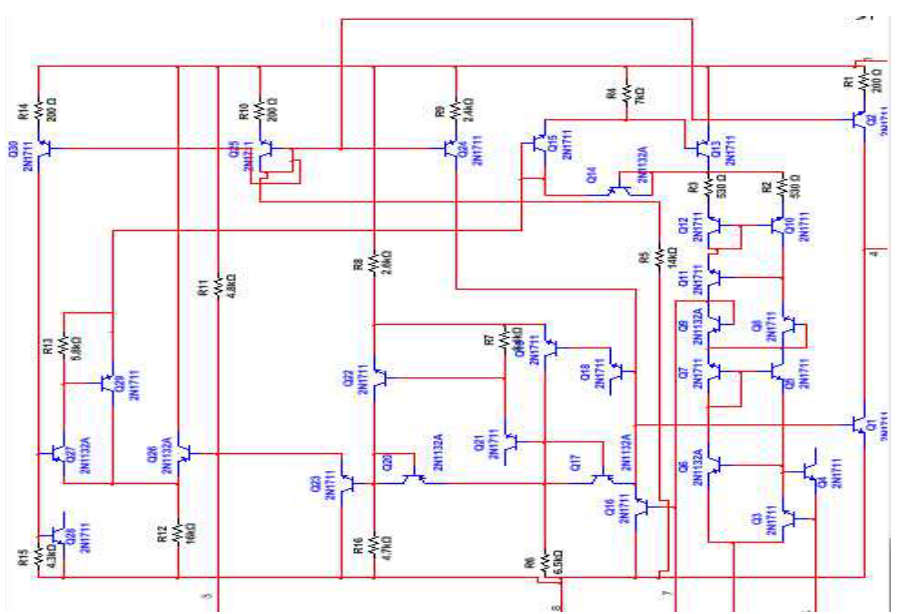

Fig.2. LM566 simulation model

\section{Simulation Analysis of Circuit Performance}

\section{Creation of simulation platform}

1) Software applied in platform Multisim12 of American NI Company; [5]

2) Components for simulation platform include resistance, capacitance, rheostat, audion (PNP and NPN) and LM566;

3) Simulation platform test instrument and parameters include:

a. Function signal generator. Regulate parameters according to need.

b. Frequency counter (frequency meter). Sensitivity and trigger electricity average are both $1 \mathrm{~V}$.

c. Dual-trace oscilloscope. Regulate parameters according to need.

d. Universal meter. Regulate it to voltage range.

Fig.3 gives schematic simulation diagram of Multisim FM cicrcuit.

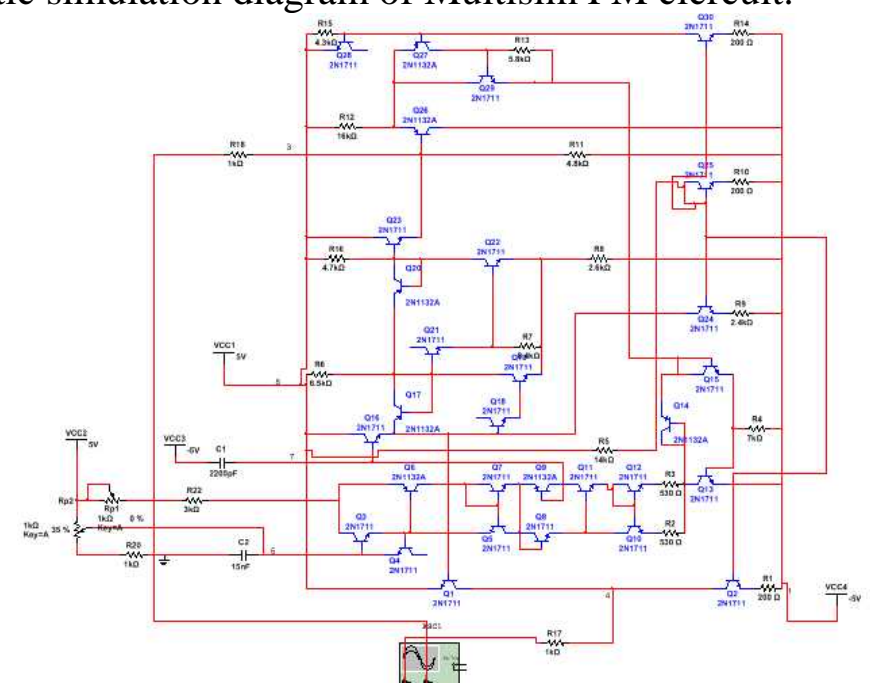

Fig.3. Multisim platform circuit diagram

\section{Simulation Analysis of circuit performance}

\section{1) Characteristics of static output frequency}

Connect LM566 pin (6) with resistance externally and connect pin (7) with capacitance externally to measure impact on frequencies of square wave and triangular wave output from pin (3) and pin (4) respectively:

Connect 2200pF capacitance $C_{1}$ with pin (7) and connect $R_{p} 2$ and $15 \mathrm{nF}$ capacitance $C_{2}$ with pin (5). Regulate $R_{p} 2$ to make $V_{5}=3.5 \mathrm{~V}$. Connect frequency meter with pin (3). Record output frequencies when $R_{T}$ (total resistance value externally connected with pin (6), similar 
hereinafter) when $R_{T}$ is at maximum and minimum. Compare the output frequency with theoretical value and use dual-trace oscilloscope to record output waveforms of square wave and triangular wave when $R_{T}$ is at minimum. [6]

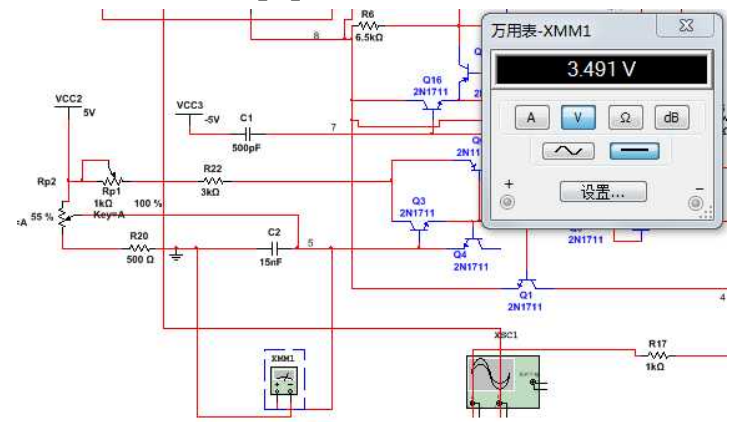

Fig.4. Regulate Rp2 to make V5=3.5V

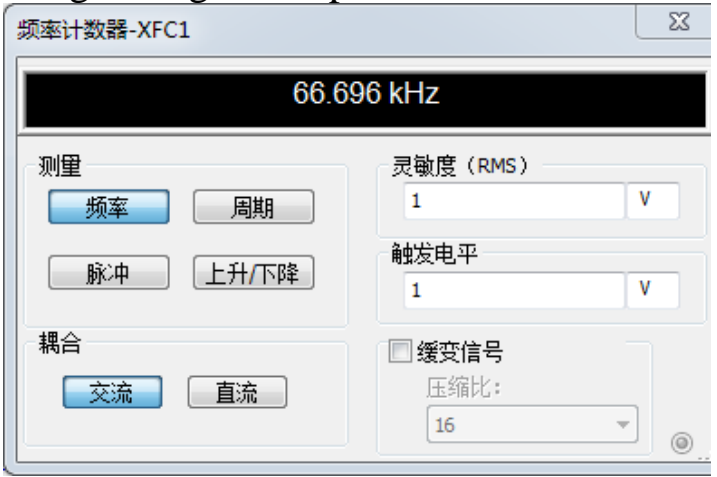

Fig.5. Output Frequency When $R_{T}$ at Maximum

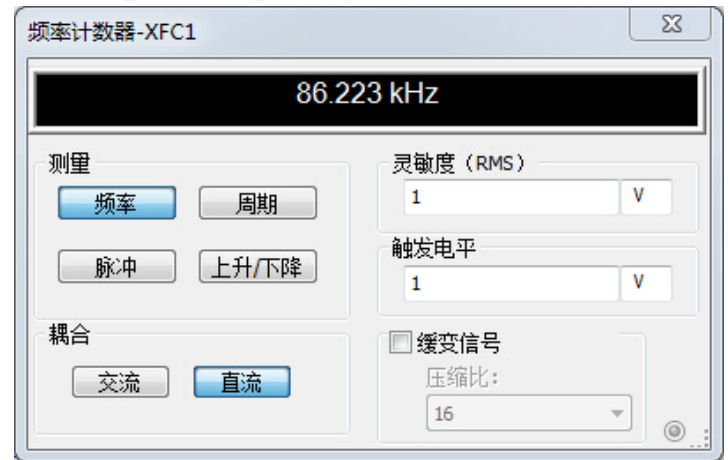

Fig.6. Output frequency when $R_{T}$ at minimum

Simulation result is basically consistent with theoretical analysis through calculation.

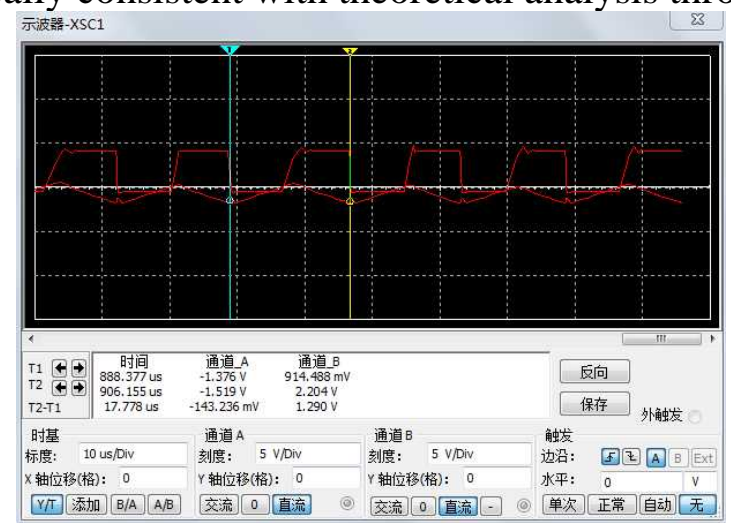

Fig.7. Output waveforms of square wave and triangular wave when $R_{T}=\mathrm{Rmin}$

2) Control of direct voltage

Regulate $R_{p} 1$ to the maximum and then change $R_{p} 2$ to regulate input voltage. Measure the change of output frequency f when $V_{5}$ changes in 2.2-4.2V. $V_{5}$ is increased progressively by $0.2 \mathrm{~V}$. Chart 1 shows the output frequencies corresponding to different control voltages. 
Chart 1 Frequency Changing with Voltage

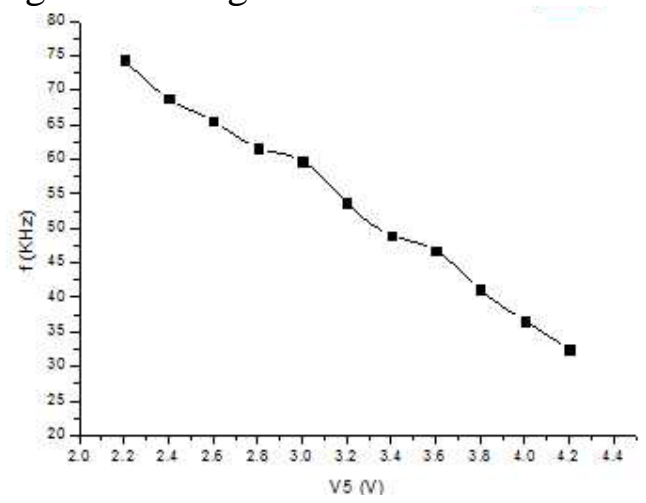

Fig.8. Relation graph of $V_{5}$ and $\mathrm{f}$

It can be concluded from Fig.8 that output frequency is decreased constantly with the increase of input voltage.

3) Control of alternating voltage

Regulate $R_{p} 1$ to maximum. Cut off $C_{2}$ connected with pin (5). Output of input signal circuit is connected with pin (5) as shown in Fig.9.

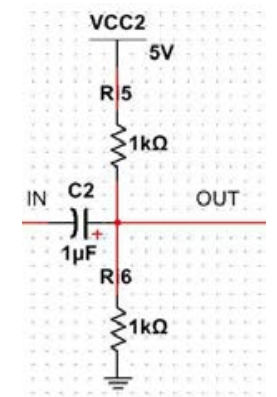

Fig.9. Input Signal Circuit

First set modulated signal em (input modulated signal) of sine wave of function signal generator $\mathrm{f}=5 \mathrm{KHz}$ and $\mathrm{Vp}-\mathrm{p}=1 \mathrm{~V}$. Then connect to IN terminal of input circuit. Use dual-trace oscilloscope to observe input signal em and output signal of frequency modulation (FM) square wave of 566 pin (3). Observe and record how waveform will be change when input signal amplitude Vp-p and frequency $f_{m}$ are changed slightly.

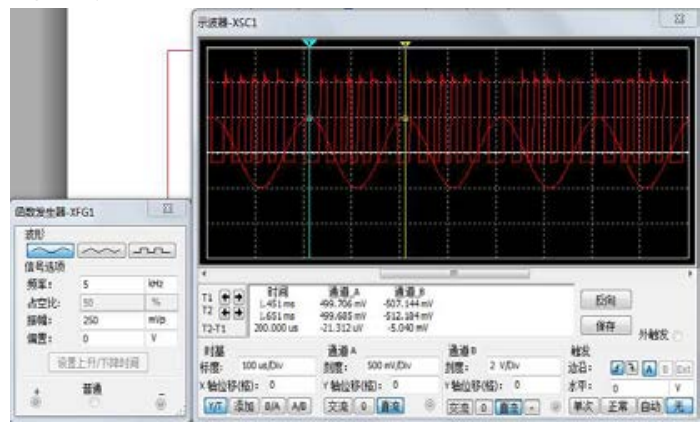

Fig.10. Output signal of fm square wave of input signal em and 566 pin

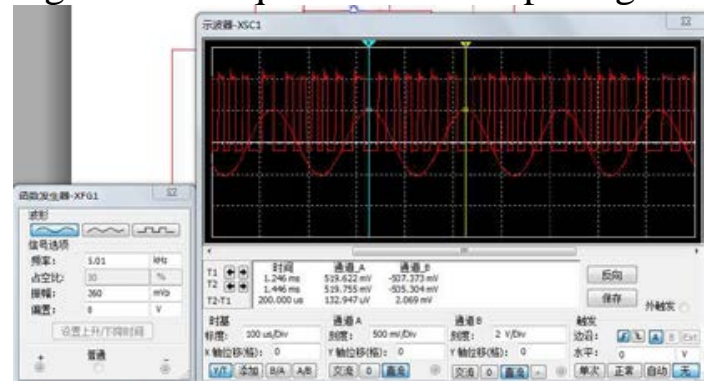

Fig.11. Change of output waveform when input signal amplitude Vp-p and frequency $f_{m}$ changed slightly 
It shows that waveform of square wave FM signal is relatively dense when modulated signal is at minimum and it is relatively sparse at maximum. It is the feature of FM square wave signal, which means modulated signal frequency is controlled by modulated signal. When modulated signal is at high level, FM signal frequency is relatively large and forward frequency offset is the maximum; when modulated signal is at low level, FM signal frequency is the least and forward frequency offset is the minimum. [10][11][12]

Change modulated signal inform square wave signal em and make frequency $f_{m}=1 \mathrm{KHz}$ and $\mathrm{Vp}-\mathrm{p}=0.35 \mathrm{~V}$. Use dual-trace oscilloscope to observe and record em and frequency modulation (FM) square wave output signal of pin (3) as shown in Fig.11.

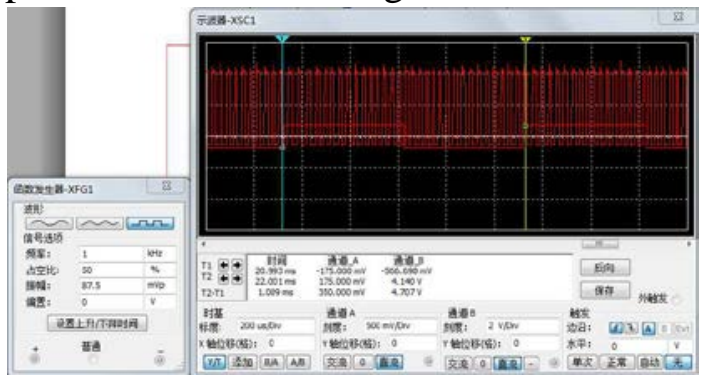

Fig.12. Output signal of frequency modulation (FM) of em and 566 pin (3)

It is shown in figure above that frequency change of FM square wave is not obvious. Change modulated signal and change Vp-p into $1 \mathrm{~V}$ and the result is as shown in Fig.12.

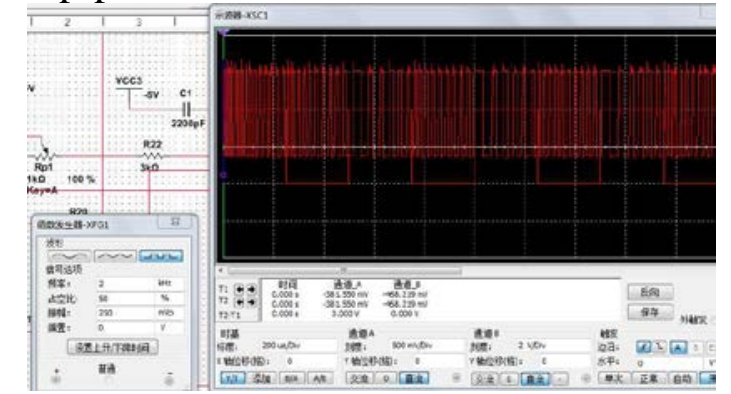

Fig.13. Observe change by changing voltage

It is obvious that FM signal frequency is controlled by modulated signal. FM signal frequency is relatively low when modulated signal is at high level and the frequency is relatively high when modulated signal is at low level. [12][13]

It can be shown in the simulation above that FM signal frequency is controlled by modulated signal. When modulated signal is at high level, FM signal frequency is relatively large and forward frequency offset is the maximum; when modulated signal is at low level, FM signal frequency is the least and forward frequency offset is the minimum. Waveform of square wave FM signal is relatively dense when modulated signal is at minimum and it is relatively sparse at maximum. [15]

\section{Conclusion}

Internal circuit diagram of LM566 chip created on Multisim simulation platform verifies electrical characteristics in FM circuit from simulation results of static output characteristic and dynamic output characteristics, which are basically consistent with indexes on datasheet and have great application value.

Use Multisim software to design circuit just like to lap circuit on the bread board in laboratory, which is not restricted by varieties and quantity of components. [16][17]The designer shall learn continuously to grasp the essence in the learning and operation of the software. We shall summarize and accumulate experience in the learning and application. 


\section{References}

[1] Jinyu $\mathrm{Hu}$ and Zhiwei Gao. Distinction immune genes of hepatitis-induced heptatocellular carcinoma[J]. Bioinformatics, 2012, 28(24): 3191-3194.

[2] Su T, Wang W, Lv Z, et al. Rapid Delaunay triangulation for randomly distributed point cloud data using adaptive Hilbert curve[J]. Computers \& Graphics, 2016, 54: 65-74.

[3] Gu W, Lv Z, Hao M. Change detection method for remote sensing images based on an improved Markov random field[J]. Multimedia Tools and Applications, 2015: 1-16.

[4] Lv Z, Tek A, Da Silva F, et al. Game on, science-how video game technology may help biologists tackle visualization challenges[J]. PloS one, 2013, 8(3): 57990.

[5] Chen Z, Huang W, Lv Z. Towards a face recognition method based on uncorrelated discriminant sparse preserving projection[J]. Multimedia Tools and Applications, 2015: 1-15. 